\title{
A Giant Mammary Hamartoma in a Young Breast Cancer Patient
}

\author{
Anna-Lena Rumpf ${ }^{a} \quad$ Micaela Mathiak $^{b}$ Fritz K. Schäfer ${ }^{c} \quad$ Almuth Caliebe ${ }^{d}$ \\ Andre Farrokh $^{a}$ Mohamed Elessawy ${ }^{a}$ Dirk O. Bauerschlag ${ }^{a} \quad$ Nicolai Maass ${ }^{a}$ \\ Marion van Mackelenbergh ${ }^{a}$ Thorsten Heilmann ${ }^{a}$ \\ ${ }^{a}$ Department of Gynecology and Obstetrics, University Hospital Schleswig-Holstein, Campus Kiel, Kiel, Germany; \\ ${ }^{b}$ Institute of Pathology, Christian-Albrechts University of Kiel, Kiel, Germany; ${ }^{\mathrm{C}}$ Department of Breast Imaging and \\ Interventions, University Hospital Schleswig-Holstein, Campus Kiel, Kiel, Germany; ${ }^{\mathrm{d} I n s t i t u t e}$ of Human Genetics, \\ University Hospital Schleswig-Holstein, Campus Kiel, and Christian-Albrechts University, Kiel, Germany
}

\section{Established Facts}

- Hamartomas are rare benign tumors of the breast, which might reach a considerable size.

- Preoperative differentiation from other benign or malignant tumors is challenging.

- The PTEN hamartoma tumor syndrome increases the risk of breast cancer.

\section{Novel Insights}

- Giant hamartomas should be removed to avoid long-range deformity of the breast.

- The presence of a mammary hamartoma might indicate the necessity of a genetic testing.

\section{Keywords}

Hamartoma of the breast · Breast cancer · PTEN mutation

\begin{abstract}
Background: Hamartomas of the breast are rare benign tumors. Pre- and also postoperative differentiation from other benign or even malignant tumors is challenging. Case Presentation: A 36-year-old female presented with a giant tumor of the left breast. The patient had suffered from an early breast cancer of the contralateral right breast the year before, which was treated with breast-conserving therapy, radiation, and endocrine therapy ever since. The hamartoma was classified as BI-RADS 2 in mammography and BI-RADS 4 in ultrasound. On clinical examination, a tumor of nearly 15
\end{abstract}

$\mathrm{cm}$ in size led to an abstruse deformity of the breast and the nipple-areola complex. We found an indolent, grand bulging tumor with an elastic texture directly beneath the skin. A biopsy that had been performed before was compatible with the suspected hamartoma. Because of the remaining diagnostic uncertainties after contralateral breast cancer and the progressive malformation of the left breast, a tumor extirpation utilizing a reduction mammaplasty was performed without complications. Subsequent genetic analyses excluded a loss of PTEN in this patient. Conclusion: We presented the rare case of a 36-year-old woman with a history of breast cancer and a 700-g breast hamartoma. The preoperative and even the postoperative specification of a hamartoma remains challenging, and associations with genetic alterations should be considered.

(c) 2020 S. Karger AG, Base 


\section{Introduction}

Breast tumors among young women mainly comprise benign lesions, such as fibroadenomas, cysts, papillomas, localized mastopathy, lipomas, phylloides tumors, whereas breast cancers are seldom events [1]. The pathogenesis of the rare breast hamartoma is still unclear. Hamartomas vary in size, clinical appearance, and histological features upon pathological examination, and patients of all ethnicities typically present with a unilateral growth of the breast [2]. Hamartomas can be detected in mammography as a pathognomonic homogeneous mass with varying ray densities, but, due to its sonolucency, its size is typically underestimated in ultrasound [3]. No defining amount of epithelial elements, adipose tissue, muscle cells, and stromal hyperplasia is described, which makes the pathological report challenging [2]. As a consequence, fine-needle biopsies may exclude malignancy, but often remain unspecific. No recurrence is seen after surgical removal.

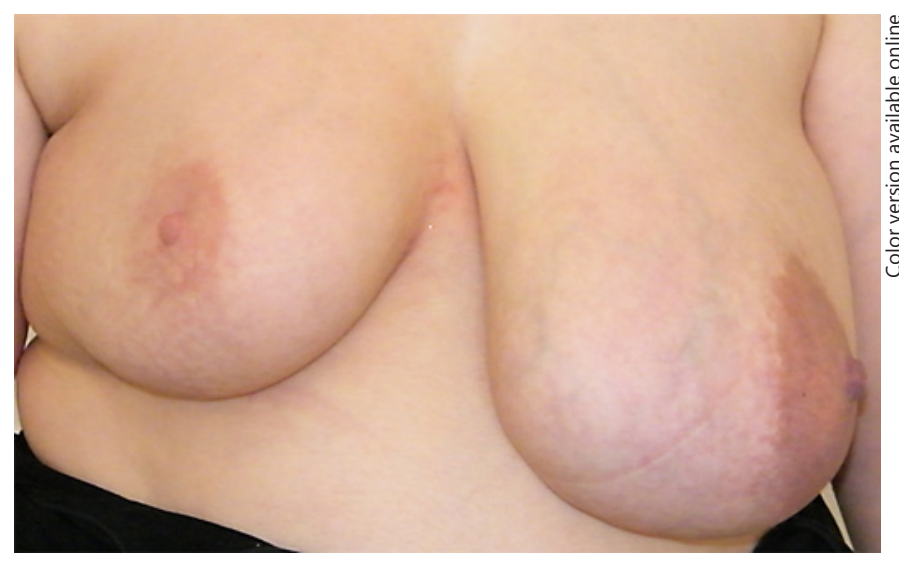

Fig. 1. Clinical presentation of a giant malformation of the left breast with an enlarged nipple-areola complex.

\section{Case Presentation}

History

A 36-year-old, healthy female patient presented with a giant palpable mass of the left breast. No history of cancer diseases in her family was reported. According to the patient's history, the enlargement of the left breast had started while being pregnant with her first child 6 years earlier. One year before, she had additionally noticed a tumor in the right, contralateral breast while breastfeeding her second child. This palpable mass in the right breast turned out to be a mucinous, hormone receptor-positive and node-negative breast cancer of $34 \mathrm{~mm}$ in size. Subsequent breast cancer therapy included breast-conserving operation on the right breast, radiation, and adjuvant endocrine therapy until recent presentation to our unit for the removal of the slowly growing mass in the left breast.

\section{Clinical Examination}

The initial physical examination showed a grand, indolent, and elastic partially cystic malformation of the left breast with a noticeable enlargement of the nipple-areola complex and signs of venous congestion. There were no signs of nipple secretion. No suspect axillary lymph nodes were detected. The volume of the left breast nearly doubled the volume of the contralateral side. Accordingly, the jugulum-nipple distance was $27 \mathrm{~cm}$ compared to $18 \mathrm{~cm}$ on the right breast (Fig. 1).

\section{Breast Imaging and Biopsy}

The tumor in the enlarged left breast qualified as a BI-RADS 2 finding in mammography, describing an encapsulated, partially radiolucent mass with a size of $15 \times 14 \times 14 \mathrm{~cm}$ pathognomonic for a hamartoma (Fig. 2). Mammary ultrasound described difficult imaging conditions with hypoechoic structures, marked duct ectasias and diffuse shadowing, thereby distinctly underestimating the size of the tumor (Fig. 2). Although the examined area did not feature any further signs of malignancy, a summarized classification as BI-RADS 4 was assigned. Consecutive core needle biopsy showed tissue with fibro-mastopathy but could not confirm the finding of a hamartoma diagnosis.

\section{Tumor Resection}

A tumor resection was indicated because of the critical deformity of the left breast and remaining diagnostic uncertainties. Tumor resection was performed via a reduction mammoplasty utilizing a superomedial pedicle technique for the nipple-areolar complex, including an inverted T-scar incision. Intraoperatively, the

Fig. 2. Mammography showing pathognomonic features on the left. Mammary ultrasound with a tumor size of $3.9 \times 2 \times 1 \mathrm{~cm}$ on the right.

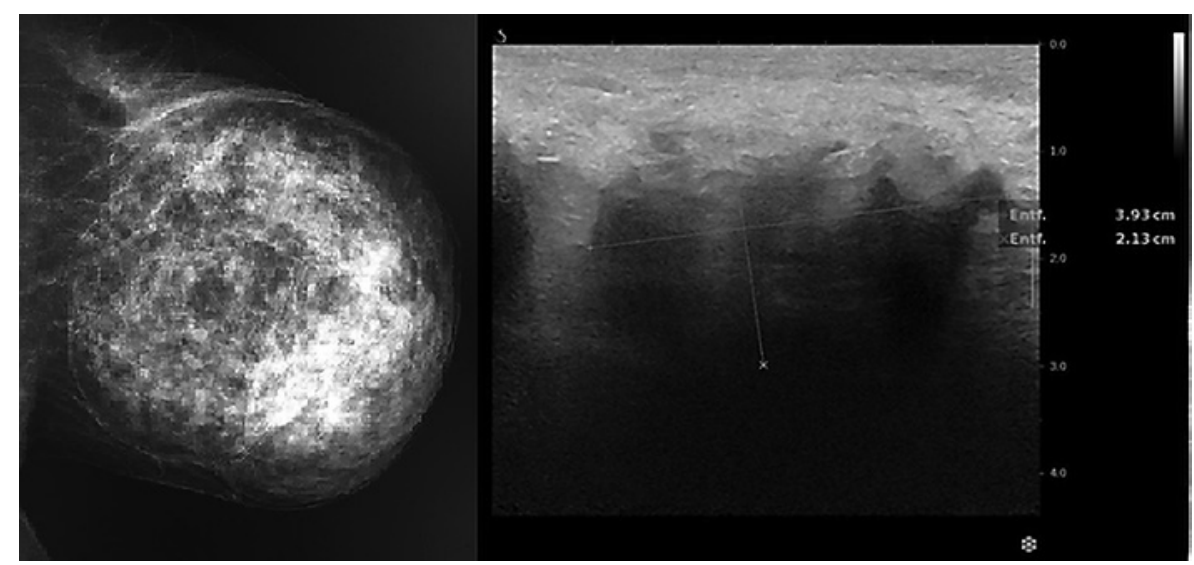


tumor was easily dissected along a capsule and removed from the surrounding breast parenchyma after ligation of a single vascular pedicle (Fig. 3). The areola complex was preserved and reduced in adaption to the right breast. A transient venous congestion of the nipple occurred after the operation, which was explained by very thin resection margins behind the nipple-areola complex. Upon follow-up examination after 14 days, the nipple already recovered and the breast showed a favorable symmetric clinical aspect (Fig. 4).

\section{Histology}

On gross examination, the tumor measured $13.4 \times 5.2 \times 15.2$ $\mathrm{cm}$ with a total weight of almost $700 \mathrm{~g}$. The tumor was well defined, being encompassed by a thin capsular structure, and showed fatty tissue mixed with fibrous strands. Within the fibrous tissue slit-like or cystic ductal structures showed congested secretion and minor calcifications. Histologically, the tumor showed fibrolipous tissue with encompassed lobular and ductal elements. There were no seminal features to distinguish from mastopathy (Fig. 5).

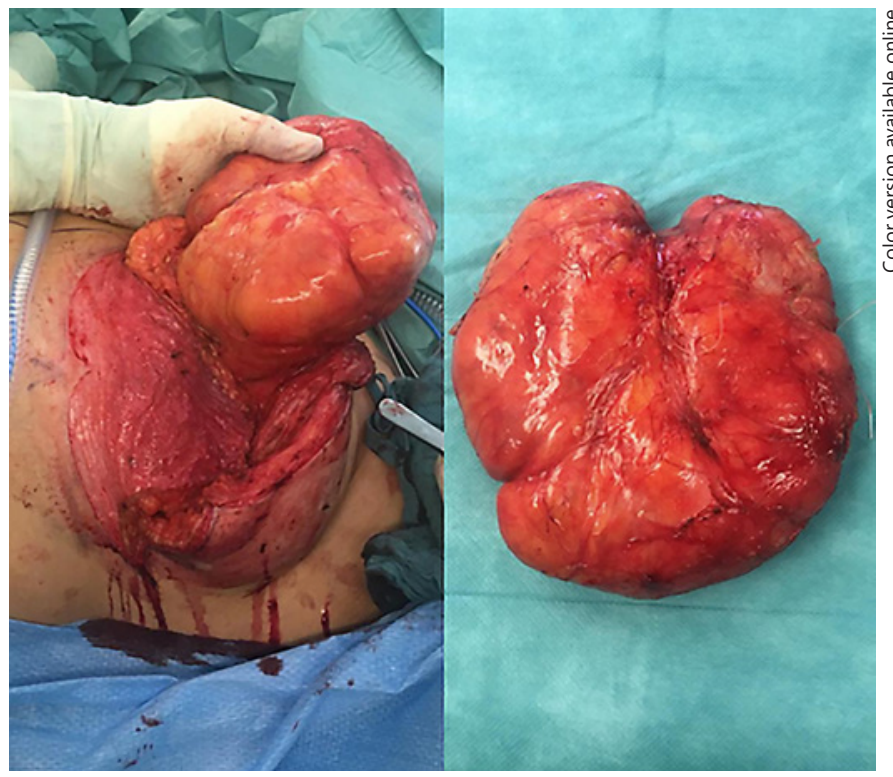

Fig. 3. Intraoperative tumor resection (left) and removed tumor (right) are shown.

\section{Discussion}

A mammary hamartoma is a rare benign tumor. Because of the limited number of cases, most publications found are case studies $[4,5]$. Per definition, a hamartoma represents a focal malformation that resembles a neoplasm, but results from a faulty development in an organ. It is composed of an abnormal mixture of normal tissue elements, or an abnormal proportion of a single element at that site. Herbert et al. [2] demonstrated that hamartomas express estrogen and progesterone receptors and exhibit an increased proliferative activity. Hamartomas might remain undetected in clinical examination and imaging because they hardly differ from normal breast tissue in ultrasound $[2,5]$.

In pathological evaluation, the diagnosis of a hamartoma can be challenging due to different quantities of the

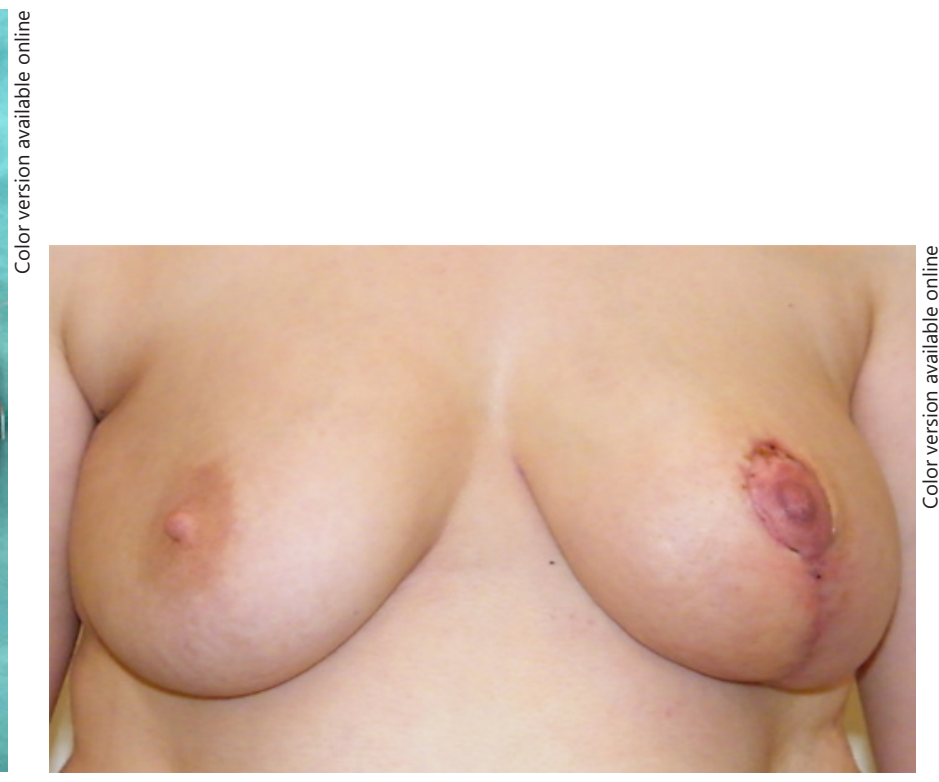

Fig. 4. Clinical presentation at the 2-week follow-up.

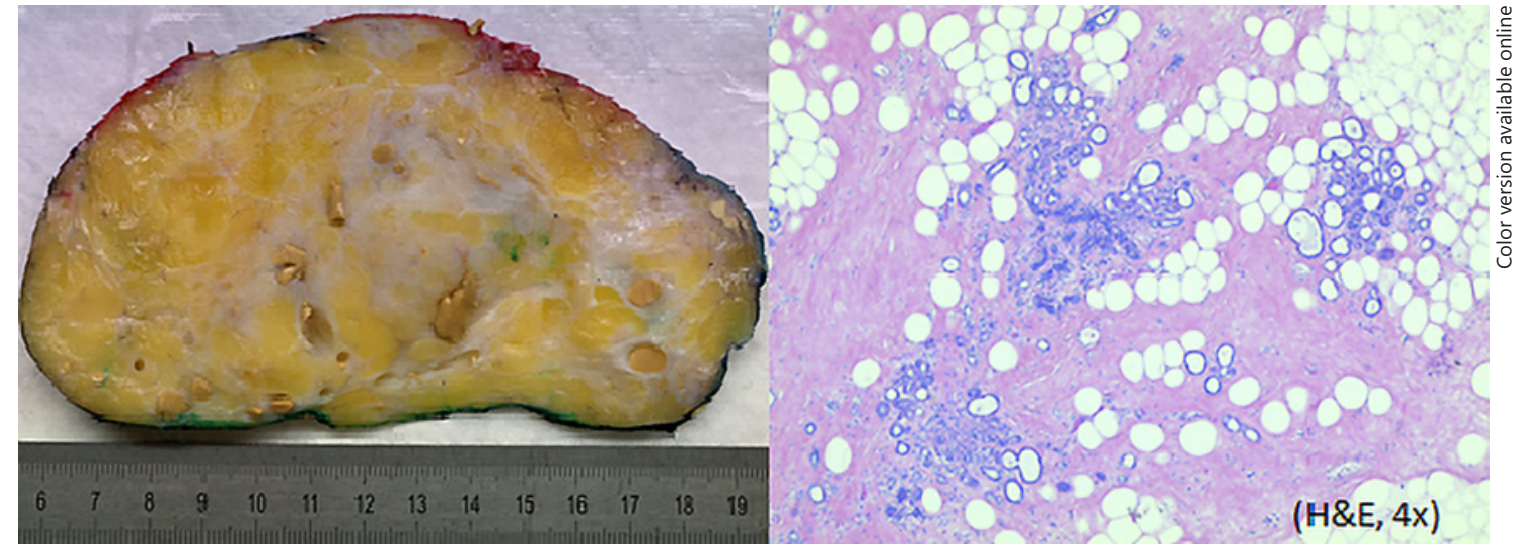

Fig. 5. Gross histological examination (left) and histological evaluation of the tumor (right) are shown. 
components. Whereas the lobular structure of the breast is normally preserved, the number of epithelial elements, fat, or stroma hyperplasia varies significantly $[2,6]$. The most common variants of hamartoma comprise adenolipoma, with their subentities fibroadenolipoma or lipofibroadenoma [7], as well as chondrolipoma. Regarding a relevant differential diagnosis of a hamartoma, primarily the fibroadenoma of the breast is to be mentioned, being the most common benign tumor among females $[8,9]$. If compared histologically, fibroadenomas present with a typical nodular fibrous stromal component encompassing variably compressed ductular structures, whereas hamartomas show typical mammary tissue arranged in a disorderly fashion [2]. In resection specimens, this differential diagnosis is usually easy to accomplish, but in needle biopsies, the differentiation can be difficult to impossible. Hamartomas with a lot of adipose tissue tend to be misinterpreted as lipomas, if the glandular component is not captured in needle core biopsy [2]. Another palpable fibroepithelial tumor of the breast, the phylloides tumor, can usually be ruled out microscopically due to the lack of increased cellularity in the stromal components in hamartomas [10].

A hamartoma itself does not classify as a marker for an increased relative risk for developing breast cancer [11]. But importantly, there is an unveiled genetic pathology linking multiple or bilateral hamartomas to breast cancer. The PTEN hamartoma tumor syndrome describes a mutation in the PTEN tumor suppressor gene, leading to a drastic increase in breast cancer lifetime risk of $67-85 \%$ and development of hamartomas in different tissues of the body $[12,13]$. Therefore, our patient was advised to consult an interdisciplinary human geneticist. Here, the genetic testing excluded a loss of PTEN function.

\section{Conclusion}

In summary, we presented a rare case of a 36-year-old woman with a giant hamartoma of the left breast in parallel to a contralateral breast cancer. Surgical tumor resection was performed based on diagnostic uncertainties and a progressive deformation of the breast. Importantly, the presence of hamartomas might indicate a genetic alteration increasing lifetime breast cancer risk. Therefore, genetic testing should be considered.

\section{Statement of Ethics}

This paper adheres to the law of Data Protection and was conducted ethically in accordance with the World Medical Association Declaration of Helsinki. The patient has given written informed consent to publish her case including publication of images. The study was additionally approved by the institute's committee on human research (D 580/19).

\section{Disclosure Statement}

The authors have no conflicts of interest to declare.

\section{Funding Sources}

No funding sources have been used.

\section{Author Contributions}

A.-L.R. and T.H. treated the patient, designed the study, and wrote the manuscript. M.M. performed histological evaluation and pathologic consulting. F.K.S. and A.F. performed imaging and radiologic consulting. A.C. performed genetic analyses. M.E., D.O.B., N.M., and M.v.M. were involved in treatment of the patient and edited the manuscript.

\section{References}

1 Paepke S, Metz S, Brea Salvago A, Ohlinger R. Benign Breast Tumours - Diagnosis and Management. Breast Care (Basel). 2018 Dec;13(6): 403-12.

2 Herbert M, Sandbank J, Liokumovich P, Yanai O, Pappo I, Karni T, et al. Breast hamartomas: clinicopathological and immunohistochemical studies of 24 cases. Histopathology. 2002 Jul; 41(1):30-4.

3 Presazzi A, Di Giulio G, Calliada F. Breast hamartoma: ultrasound, elastosonographic, and mammographic features. Mini pictorial essay. J Ultrasound. 2015 Jul;18(4):373-7.

4 Charpin C, Mathoulin MP, Andrac L, Barberis J, Boulat J, Sarradour B, et al. Reappraisal of breast hamartomas. A morphological study of 41 cases. Pathol Res Pract. 1994 Apr;190(4):362-71.
5 Sevim Y, Kocaay AF, Eker T, Celasin H, Karabork A, Erden E, et al. Breast hamartoma: a clinicopathologic analysis of 27 cases and a literature review. Clinics (São Paulo). 2014 Aug;69(8):515-23.

6 Fisher CJ, Hanby AM, Robinson L, Millis RR. Mammary hamartoma-a review of 35 cases. Histopathology. 1992 Feb;20(2):99-106.

7 Altermatt HJ, Gebbers JO, Laissue JA. Multiple hamartomas of the breast. Appl Pathol. 1989; 7(2):145-8.

8 Krings G, Bean GR, Chen YY. Fibroepithelial lesions; The WHO spectrum. Semin Diagn Pathol. 2017 Sep;34(5):438-52.

9 Arca MJ, Caniano DA. Breast disorders in the adolescent patient. Adolesc Med Clin. 2004 Oct;15(3):473-85.
10 Zhang Y, Kleer CG. Phyllodes Tumor of the Breast: Histopathologic Features, Differential Diagnosis, and Molecular/Genetic Updates. Arch Pathol Lab Med. 2016 Jul;140(7):665-71.

11 Tse GM, Law BK, Pang LM, Cheung HS. Ductal carcinoma in situ arising in mammary hamartoma. J Clin Pathol. 2002 Jul;55(7):541-2.

12 Ngeow J, Sesock K, Eng C. Breast cancer risk and clinical implications for germline PTEN mutation carriers. Breast Cancer Res Treat. 2017 Aug;165(1):1-8.

13 Bubien V, Bonnet F, Brouste V, Hoppe S, Barouk-Simonet E, David A, et al.; French Cowden Disease Network. High cumulative risks of cancer in patients with PTEN hamartoma tumour syndrome. J Med Genet. 2013 Apr;50(4):255-63. 